\title{
Are psychiatric disorders risk factors for COVID-19 susceptibility and severity? a two-sample, bidirectional, univariable, and multivariable Mendelian Randomization study
}

\author{
Jurjen J. Luykx $\mathbb{D}^{1,2,3}$ and Bochao D. Lin ${ }^{1,3,4}$
}

\begin{abstract}
Observational studies have suggested bidirectional associations between psychiatric disorders and COVID-19 phenotypes, but results of such studies are inconsistent. Mendelian Randomization (MR) may overcome the limitations of observational studies, e.g., unmeasured confounding and uncertainties about cause and effect. We aimed to elucidate associations between neuropsychiatric disorders and COVID-19 susceptibility and severity. To that end, we applied a two-sample, bidirectional, univariable, and multivariable MR design to genetic data from genome-wide association studies (GWASs) of neuropsychiatric disorders and COVID-19 phenotypes (released in January 2021). In single-variable Generalized Summary MR analysis, the most significant and only Bonferroni-corrected significant result was found for genetic liability to BIP-SCZ (a combined GWAS of bipolar disorder and schizophrenia as cases vs. controls) increasing risk of COVID-19 (OR $=1.17,95 \% \mathrm{Cl}, 1.06-1.28)$. However, we found a significant, positive genetic correlation between BIP-SCZ and COVID-19 of 0.295 and could not confirm causal or horizontally pleiotropic effects using another method. No genetic liabilities to COVID-19 phenotypes increased the risk of (neuro)psychiatric disorders. In multivariable MR using both neuropsychiatric and a range of other phenotypes, only genetic instruments of BMI remained causally associated with COVID-19. All sensitivity analyses confirmed the results. In conclusion, while genetic liability to bipolar disorder and schizophrenia combined slightly increased COVID-19 susceptibility in one univariable analysis, other MR and multivariable analyses could only confirm genetic underpinnings of BMI to be causally implicated in COVID-19 susceptibility. Thus, using MR we found no consistent proof of genetic liabilities to (neuro) psychiatric disorders contributing to COVID-19 liability or vice versa, which is in line with at least two observational studies. Previously reported positive associations between psychiatric disorders and COVID-19 by others may have resulted from statistical models incompletely capturing BMI as a continuous covariate.
\end{abstract}

\section{Introduction}

Several large population-based studies have investigated associations between positive testing for COVID-19 on the one hand and psychiatric disorders on the other ${ }^{1-3}$.

\footnotetext{
Correspondence: Jurjen J. Luykx (j.luykx@umcutrecht.nl)

'Department of Psychiatry, UMC Utrecht Brain Center, Utrecht University, Utrecht, The Netherlands

${ }^{2}$ Second opinion outpatient clinic, GGNet Mental Health, Warnsveld, The Netherlands

Full list of author information is available at the end of the article
}

Positive test result likelihoods for psychiatric disorders are inconsistent between those studies: while two of those cohort studies (from the UK and South Korea) do not report positive associations between COVID-19 testing and psychiatric disorders ${ }^{1,4}$, others (from the UK and the US) mention odds ratios of $1.5-10$ for associations between mental disorders and a COVID-19 diagnosis ${ }^{2,3,5}$. One observational study reports bidirectional associations between psychiatric diagnosis in the previous year and COVID- $19^{2}$. For a recent diagnosis of a mental disorder in

\section{(c) The Author(s) 2021}

\footnotetext{
(c) Open Access This article is licensed under a Creative Commons Attribution 4.0 International License, which permits use, sharing, adaptation, distribution and reproduction cc) in any medium or format, as long as you give appropriate credit to the original author(s) and the source, provide a link to the Creative Commons license, and indicate if changes were made. The images or other third party material in this article are included in the article's Creative Commons license, unless indicated otherwise in a credit line to the material. If material is not included in the article's Creative Commons license and your intended use is not permitted by statutory regulation or exceeds the permitted use, you will need to obtain permission directly from the copyright holder. To view a copy of this license, visit http://creativecommons.org/licenses/by/4.0/.
} 
the US ${ }^{2}$, odds ratios for COVID-19 were reported to be around 7.6, with evidence for relatively severe COVID-19 outcomes in those with a diagnosis of mental disorder 5 . One limitation of these studies is that psychiatric diagnoses were grouped by some ${ }^{2,4}$, e.g., as mental disorders, psychotic disorders or affective disorders, precluding conclusions about COVID-19 risks for specific psychiatric disorders. In addition, some diagnoses, such as bipolar disorder (BIP), were not included in some of the analyses ${ }^{3}$. Furthermore, correction for medical comorbidities decreased several high odd ratios ${ }^{5}$. Finally, some authors admit residual socioeconomic factors may not be sufficiently captured in some databases ${ }^{2}$. Indeed, as recently noted, confounding or biases (partly) explain associations of COVID-19 with a range of traits and diseases ${ }^{6}$. Mendelian Randomization (MR) has the potential to overcome two major limitations of observational studies: unmeasured confounding and uncertainties about cause and effect. Examples of MR studies elucidating risk factors for COVID-19 include two recent studies showing that body mass index (BMI) and smoking are risk factors for COVID-197,8. We are not aware of preprinted or published MR studies of psychiatric disorders and COVID-19. The recently updated whole-genome data on COVID-19 phenotypes (https://www.covid19hg.org/results/, see "Methods" section) further increases the timeliness of MR approaches to elucidate risk factors for COVID-19 diagnosis and severity.

We hypothesized that given the aforementioned inconsistent observational evidence and attenuation of reported odds ratios when including covariates, psychiatric disorders do not constitute strong risk factors to contract COVID-19 or develop a severe course of COVID-19. Similarly, we hypothesized that genetic liability to COVID-19 would not increase susceptibility to psychiatric disorders. To test our hypotheses, we performed a range of bidirectional univariable and multivariable MR (MVMR) analyses of genetic liability to major psychiatric disorders and to COVID-19 susceptibility as well as severe COVID-19.

\section{Methods}

\section{Overview}

We performed two-sample MR using summary statistics from large genome-wide association study (GWASs) to examine whether genetic liabilities to (neuro)psychiatric disorders increase the risk of contracting COVID-19 and of a severe course of COVID-19 (forward MR analyses, considering (neuro)psychiatric disorders as exposures and COVID-19 as outcome). In addition, we used COVID-19 GWASs to examine the potential effects of genetic liabilities to COVID-19 diagnosis and to COVID19 severity on (neuro)psychiatric diseases (reverse MR analyses, considering COVID-19 as the exposure and (neuro)psychiatric phenotypes as the outcomes). The principle of MR analyses is shown and further explained in Supplementary Fig. 1. Most of our methods outlined below have been previously explained in more detail ${ }^{9}$. The GWAS summary statistics we used were drawn from studies that had obtained written informed consent from participants and received ethical approval from institutional review boards. No ethical approval for the current analyses was needed as they were based on publicly available summary statistics.

\section{(Neuro)psychiatric summary statistics}

We used the available GWASs with summary statistics for psychiatric disorders including Alzheimer's dementia (AD) (a meta-analysis of the stage 1 discovery dataset ${ }^{10}$ ), anxiety $^{11,12}$, anxiety and stress-related diagnoses $(\mathrm{ASRD})^{11,12}$, major depressive disorder $(\mathrm{MDD})^{13}, \mathrm{BIP}^{14}$, schizophrenia $(\mathrm{SCZ})^{15}$, and a meta-analysis of BIP and SCZ (BIP-SCZ; Table 1$)^{16}$. To avoid that sample overlap between exposures' and outcomes' datasets impacted results substantially by inducing instrument bias in MR analyses, we excluded UK Biobank (UKBB) cohorts from (neuro)psychiatric disorders summary statistics. The largest Anorexia Nervosa GWAS and the largest 2018 and 2019 MDD GWASs contained a large number of UKBB participants, precluding us from using those GWASs for MR analyses. Hence, we selected MDD summary statistics from the 23andMe cohort $^{13}$, containing only 10,000 independent SNPs due to participants consent, and thus used these summary statistics only for univariable forward MR analysis. Similarly, we refrained from using the psychiatric cross-disorder GWAS as its study population was also partly composed of UKBB participants. For anxiety and ASRD, we performed meta-analysis in METAL ${ }^{17}$ excluding UKBB participants: of anxiety using the iPSYCH (4584 cases and 19,225 controls) and ANGST cohorts (7016 cases and 14,745 controls) and of ASRD also using the iPSYCH cohort (4584 cases and 19,225 controls) and ANGST cohorts (12,665 cases and 14,745 controls). We used the anxiety and ASRD summary statistics we had thus generated for our MR analyses ${ }^{18}$.

\section{COVID-19 summary statistics}

We used the most recent COVID-19 (GWAS) metaanalyses round $4(\mathrm{~A} 1, \mathrm{C} 1, \mathrm{D} 1$; Table 2$)$ and $5(\mathrm{~A} 2, \mathrm{~B}, \mathrm{~B} 2$, $\mathrm{C} 2$, Table 2; round 5 being an updated release from January 18,2021 , with more cohorts and larger sample sizes compared to round 4 only for phenotypes A2, B1, B2, and C2 $)^{19}$, released on October 20, 2020, and January 18, 2021, respectively, from the COVID-19 Host Genetics Initiative (https://www.covid19hg.org/results/) containing several COVID-19 phenotypes. This GWAS was based on a study population drawn from multiple cohorts, with European being the dominant ancestry: BioMe, FinnGen, Genes \& 
Table 1 GWASs of (neuro)psychiatric disorders used for the current study and outcomes used for the univariable forward MR analyses.

\begin{tabular}{|c|c|c|c|c|c|}
\hline GWASs & Cohorts & Number of loci & $\begin{array}{l}\text { Number } \\
\text { of cases }\end{array}$ & $\begin{array}{l}\text { Number of } \\
\text { controls }\end{array}$ & $\begin{array}{l}\text { Outcomes used for univariable forward MR } \\
\text { analyses (see Table } 2 \text { for definitions of A-D) }\end{array}$ \\
\hline Anxiety & iPSYCH + ANGST & 0 & 11,600 & 33,970 & \\
\hline ASRD & iPSYCH + ANGST & 1 & 19,681 & 33,970 & \\
\hline$A D$ & $\begin{array}{l}\text { ADGC, CHARGE, ADI, } \\
\text { ERAD/PERADES. }\end{array}$ & 32 & 21,982 & 41,944 & $\mathrm{~A} 1, \mathrm{~A} 2, \mathrm{~B} 1, \mathrm{~B} 2, \mathrm{C} 1, \mathrm{C} 2, \mathrm{D} 1$ \\
\hline MDD & 23andMe (10k SNPs) & 5 & 75,607 & 231,747 & $A 1, A 2, B 1, B 2, C 1, C 2, D 1$ \\
\hline BIP & PGC & 6 & 53,555 & 54,065 & $A 1, A 2, B 1, B 2, C 1, C 2, D 1$ \\
\hline $\mathrm{SCZ}$ & PGC + CLOZ-UK & 138 & 40,675 & 64,643 & $A 1, A 2, B 1, B 2, C 1, C 2, D 1$ \\
\hline BIP-SCZ & PGC + CLOZ-UK & 96 & 20,129 & 21,524 & $A 1, A 2, B 1, B 2, C 1, C 2, D 1$ \\
\hline
\end{tabular}

A1-D1 are COVID-19 phenotypes that are defined and explained in Table 2 . In bold are depicted the (neuro)psychiatric GWASs that had identified $\geq 2$ genome-wide significant loci and were thus selected as exposures in our forward MR analyses.

Anxiety anxiety disorders, ASRD anxiety and stress-related disorders, $A D$ Alzheimer's disease, MDD major depressive disorder, BIP bipolar disorder, SCZ schizophrenia, $B I P-S C Z$ a combined GWAS of bipolar disorder and schizophrenia vs. controls.

Health, LifeLines Global Screening Array, LifeLines CytoSNP, Netherlands Twin Register, Partners Healthcare Biobank, and UKBB (Table 2; Supplementary Table 1). The majority of the included subjects were of European descent, with a small proportion of other ethnic backgrounds. Only variants with imputation quality $>0.6 \mathrm{had}$ been retained and meta-analysis of individual studies had been performed with inverse variance (IV) weighting by the authors of the COVID-19 Host Genetics Initiative. To avoid that sample overlap between exposures' and outcomes' datasets impacted results substantially by inducing instrument bias in MR analyses, we only included COVID-19 summary statistics that had excluded the 23andMe cohort. We divided the COVID-19 phenotypes (A-D, Table 2) into two categories, COVID-19 susceptibility and severity. We used two phenotypes to index COVID-19 susceptibility, namely $C$ (defined by the COVID-19 Host Genetics Initiative as partial susceptibility) and D (self-reported COVID-19). Similarly, we used A (very severe respiratory confirmed COVID-19, here: 'very severe COVID-19') and B (hospitalized labconfirmed COVID-19, here 'severe COVID-19') to index COVID-19 severity.

\section{MR analyses}

Data were analyzed between November, 2020 and January, 2021. As our main analysis, we performed univariable MR using GSMR (Generalized Summary MR) ${ }^{20}$ implemented in the Genome Complex Trait Analysis (GCTA) software ${ }^{21}$. For our exposures, we first selected all relevant single-nucleotide variants (SNVs) identified in each GWASs as having reached a selection $p$ value threshold $<5 \times 10^{-8}$ and being non-duplicate and uncorrelated $(10,000 \mathrm{kbp}$ apart and Linkage Disequilibrium
(LD) $\left.R^{2} \leq 0.001\right)$. Instrument outliers were identified using HEIDI-outlier test $(p<0.01)$, with the minimum number of instruments required for the GSMR analysis $($ nsnps_thresh $)=2$. In harmonizing exposure and outcome data, we removed palindromic SNVs with intermediate allele frequencies, and SNVs with minor-allele frequency differences $>0.2$ between exposure GWASs and outcome GWASs. We estimated the F-statistic from first-stage regression to evaluate instrument strength, which is defined as the ratio of the mean square of the model to the mean square of the error. The rule of thumb is that a threshold of $F<10$ indicates weak instrument strength ${ }^{22}$.

To interpret our results, we advise readers to take into account effect sizes and not focus on $p$ values. As a cut-off for statistical significance, we Bonferroni corrected twosided $p<0.05$ for the number of tests performed in all analyses, i.e., univariable forward $(n=35)$, univariable reverse $\operatorname{MR}(n=16)$, and multivariable forward and reverse (see "Results" section).

Then, Bonferroni-corrected significant results from GSMR were validated by applying as sensitivity analyses several alternative MR models, namely fixed-effect inverse variance weighted (IVW), MR-Egger ${ }^{23}$, weighted medianbased regression methods ${ }^{20}$, and MR pleiotropy residual sum and outlier (MR-PRESSO) ${ }^{24}$ that depend on different assumptions. The harmonized input data from GSMR were used to perforby "TwoSampleMR" R packages ${ }^{25}$. For significant results, we used the MR-Egger intercept test, Cochran Q heterogeneity test, and MR-PRESSO test to evaluate potential IV violations, and

Causal Analysis Using Summary Effect estimates $(\mathrm{CAUSE})^{26}$ to estimate horizontal pleiotropy. We also performed leave-one-out analyses to examine whether 


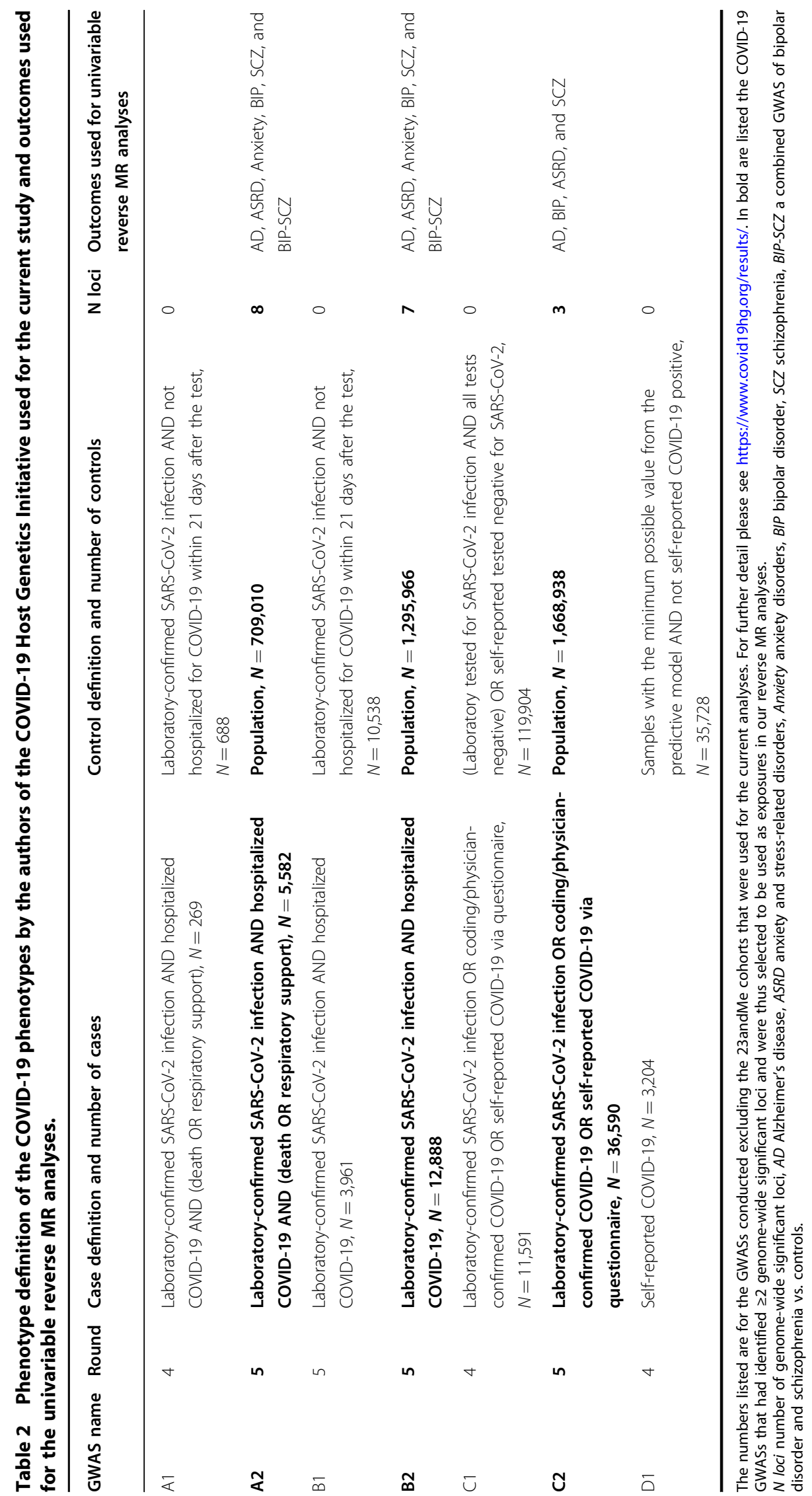


any high-impact instruments possibly influenced MR results disproportionally.

At last, we conducted MVMR analyses ${ }^{27}$ using the Mendelian Randomization $\mathrm{R}$ package to examine which phenotypes remained risk factors taking into account pleiotropic effects among exposures. MVRM estimates the effects of each exposure on an outcome adjusting for genetic associations between multiple phenotypes and the exposure. Scenarios one may think of are when a researcher hypothesizes that exposures are related to one another or when one exposure may mediate the relationship between the exposure of interest and an outcome ${ }^{28}$. MVMR does so by using genetic instruments (derived from either summary-level or individual-level data) associated with each of those multiple phenotypes. MVMR is an extension of MR that may prove more useful and reliable in scenarios where three or more exposures may be at play. Examples for the field of psychiatry include MVMR analyses for self-harm ${ }^{29}$ and $\mathrm{SCZ}^{9}$. We thus considered MVMR a useful method to follow up and corroborate our initial findings. For MVMR analyses, we constructed instruments using SNVs in each of the GWASs meeting our single-variable MR selection criteria, as described above. We used the MVMR extension of the IVW MR method and MR-Egger method to correct for measured and unmeasured pleiotropy. In forward MVMR, we used as exposures SCZ, BIP, and AD, while $\mathrm{A} 1, \mathrm{~A} 2, \mathrm{~B} 1, \mathrm{~B} 2, \mathrm{C} 1, \mathrm{C} 2$, and D1 COVID-19 phenotypes (Table 1) were the outcomes. We excluded the following (neuro)psychiatric diseases as exposures in forward MVMR: BIP-SCZ ${ }^{16}$ as it was highly correlated with SCZ and BIP (with most of the SNVs overlapping with either $\mathrm{SCZ}$ or BIP); $\mathrm{MDD}^{13}$ as it did not have enough SNV information (summary statistics containing only 10,000 independent SNPs due to participants consent); anxiety and ASRD $^{11,12}$ as they did not have enough instrument variables, i.e., $<2$. In reverse MVMR analyses, we used the three COVID-19 A2, B2, and C2 phenotypes (Table 2) as exposures since they had the required number of $\geq 2$ genome-wide significant loci, while outcomes were ASRD, AD, anxiety, BIP, SCZ, and BIP-SCZ. We removed duplicate and correlated SNVs (within 10,000 kbp; LD $R^{2} \geq 0.001$ ), resulting in 8 SNPs as COVID-19 instruments and 119 SNPs as (neuro)psychiatric instruments. Statistical significance for MVMR was Bonferroni-corrected for the number of outcomes (see "Results" section). In the end, genetic heritability and genetic correlations between the neuro(psychiatric) disorders, BMI, and COVID-19 phenotypes were estimated using LD Score Regression $(\mathrm{LDSC})^{30}$.

\section{Sensitivity analyses}

To corroborate the robustness and consistency of our findings, we performed two sensitivity analyses. First, we used a more lenient threshold of $\left(p<1 \times 10^{-7}\right)$ for genetic instruments as a few SNPs passed conventional genomewide significant level $\left(p<5 \times 10^{-8}\right)$, only explaining a small amount of the variance in the complex trait, which in turn may increase chances of type 2 error in MR. This method of relaxing the statistical threshold for genetic instruments has been used in psychiatric MR research when few significant SNPs were available ${ }^{31-33}$.

Second, we performed MVMR with more exposures since conditions, such as obesity, diabetes, and heart diseases may increase the severity of COVID-19 and these and others (e.g., educational attainment (EA) and cognitive performance $(\mathrm{CP})$ ) may be associated with (neuro) psychiatric phenotypes. To that end, we ran univariable MR analyses for COVID-19 outcomes as discussed above using the largest GWAS summary statistics for $\mathrm{BMI}^{34}$, type 2 diabetes $(\mathrm{T} 2 \mathrm{D})^{18}$, Coronary artery disease $(\mathrm{CAD})^{35}$, $\mathrm{EA}$ and $\mathrm{CP}^{36}$, and Cross-disorder psychiatric disorders (CDG, a combined GWAS of eight psychiatric phenotypes; here CDG can be used as for COVID-19 datasets UKB and 23andMe sections had been excluded; Supplementary Table 2$)^{37}$. Then the abovementioned conditions along with the phenotypes mentioned in Table 2 were entered into MVMR analysis as exposures. To avoid sample overlap influencing our results, we conducted MR using COVID-19 round 5 summary statistics which excluded both UKBB and 23andMe cohorts; namely A2, $\mathrm{B} 1, \mathrm{~B} 2$, and C2. For (neuro)psychiatric disorders, we replaced the summary statistics containing UKBB participants with $\mathrm{MDD}^{38}$ and ASRD ${ }^{39}$ GWASs that exclude 23andMe or UKBB cohorts.

\section{Results \\ Overview}

Five neuropsychiatric disorders (numbers of instruments: $\mathrm{AD}=32, \mathrm{BIP}=6, \mathrm{MDD}=5, \mathrm{SCZ}=137$, and $\mathrm{BIP}-$ $\mathrm{SCZ}=96$; total study populations: $\mathrm{AD}=63,926 ; \mathrm{BIP}=$ 198,$882 ; \mathrm{MDD}=307,354 ; \mathrm{SCZ}=105,318$; and BIP-SCZ $=$ $107,620)$ had enough $(\geq 2)$ genome-wide loci to perform forward MR analyses (Table 1) on the seven COVID-19 phenotypes defined in Table 2. Conversely, three COVID19 phenotypes (numbers of instruments: $\mathrm{A} 2=8, \mathrm{~B} 2=7$, and $\mathrm{C} 2=3$; total study populations: $\mathrm{A} 2=1,308,275, \mathrm{~B} 2=$ $969,689, \mathrm{C} 2=1,388,510$; Table 2) had enough genomewide loci to perform reverse MR analyses. As the instrument strength was strong (F-statistic in forward and reverse MR analyses ranging from 36.2 to 69.5 ), we did not find evidence of weak instrument bias.

\section{Forward MR results of (neuro)psychiatric disorders as potential risk factors for COVID-19 phenotypes}

We performed 35 univariable MR tests to examine the potential effects on COVID-19 of genetic liabilities to five psychiatric phenotypes with at least two significant loci 


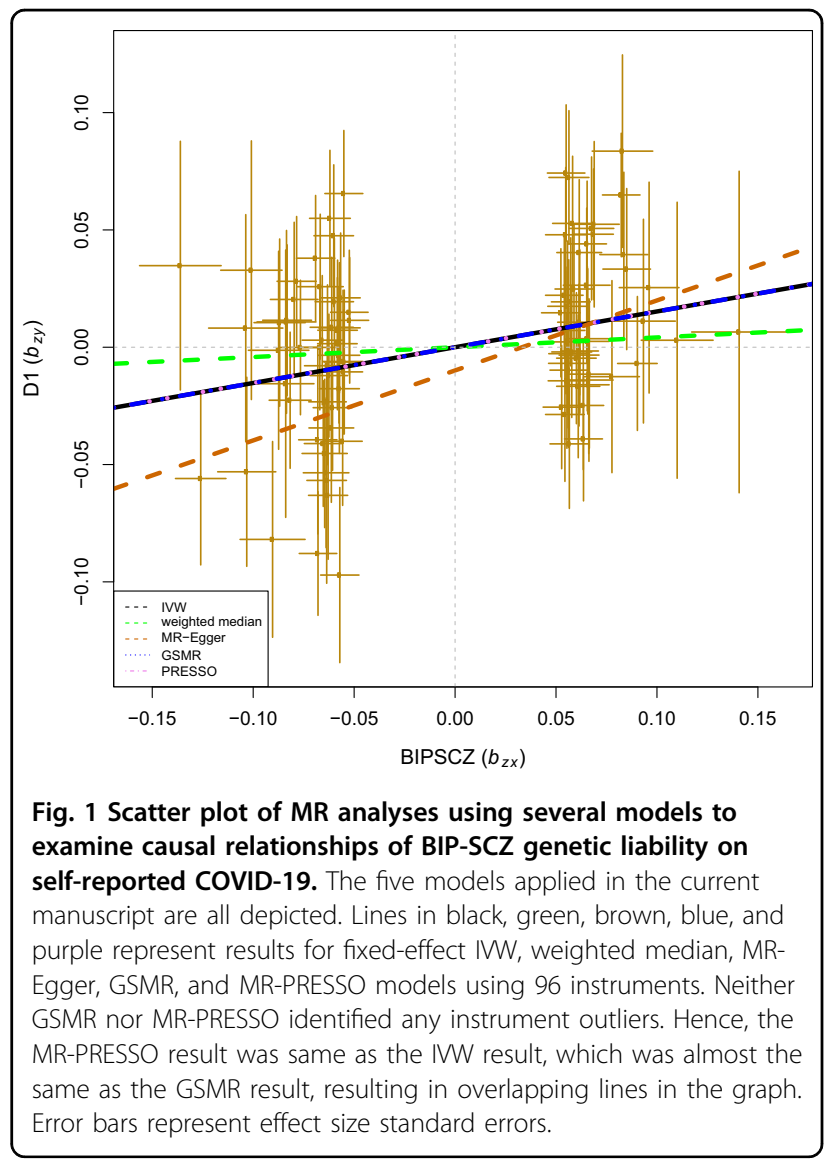

Table 3 Forward MR results of BIP-SCZ as a risk factor for self-reported COVID-19 (D1), using 96 instrument variables.

\begin{tabular}{lllll}
\hline MR model & OR & Lower $95 \% \mathrm{Cl}$ & Upper 95\% Cl & $\boldsymbol{p}$ value \\
\hline GSMR & 1.165 & 1.062 & 1.277 & 0.0012 \\
Inverse variance & 1.162 & 1.052 & 1.283 & 0.0031 \\
weighted & & & & \\
MR-PRESSO & 1.162 & 1.052 & 1.283 & 0.0045 \\
Weighted median & 1.045 & 0.91 & 1.2 & 0.5336 \\
MR-Egger & 1.349 & 0.816 & 2.23 & 0.2467 \\
\hline
\end{tabular}

identified. We thus Bonferroni corrected the significance threshold to $0.05 / 35=0.0014$. Single-variable GSMR analysis showed that the top MR result was BIP-SCZ (combined GWAS of BIP and SCZ as cases vs. controls): the effect estimate was consistent with increased COVID19 susceptibility (D1; $N=96$ instruments; OR, 1.165, 95\% CI, 1.062-1.277; $p=0.0012$; Fig. 1). All four sensitivity MR analyses confirmed the direction of effect detected by GSMR (Table 3). IVW, weighted median, and MRPRESSO also showed similar $p$ values to GSMR (Table 3). Although MR-Egger was not significant $(p=0.247)$, overall horizontal pleiotropic effects were absent (for intercept of MR-Egger: $b=0.015, p=0.293)$. The $\mathrm{I}^{2}{ }_{\mathrm{GX}}$ statistics $\left(\mathrm{I}^{2}{ }_{\mathrm{GX}}=1.07 \%\right)$ of MR-Egger were substantially smaller than $90 \%$, suggesting substantial bias in the causal estimates due to uncertainty in the genetic associations, resulting in MR-Egger results not being reliable. The Cochran's $\mathrm{Q}$ test in the fixed-effect IVW model (Q statistic $=9.395, p=0.152)$ and MR-Egger model (Q statistic $=7.278, p=0.2$ ) suggested that there was absence of heterogeneity in the instrumental variables, which may be the result of true causality rather than violation of instrument variable assumptions. Furthermore, the leaveone-out analysis (Fig. 2a) showed that no SNVs altered the pooled IVW beta coefficient, confirming the stability of our results. We also found an absence of directional pleiotropy and the instrument strength independent of direct effect assumption to be satisfied (Fig. 2b) ${ }^{23}$. As we found a genetic correlation between BPSCZ and D1 ( $\mathrm{rg}=$ $0.26, \mathrm{SE}=0.12, p=0.012$ ), horizontal pleiotropy may influence causality effects. Using CAUSE to estimate causal effects and potentially horizontal pleiotropic effects, no significant causal effects $(\gamma=0.04,95 \% \mathrm{CI}=$ -0.03 to 0.11$)$ or horizontally pleiotropic effects $(\eta=0.1$ $95 \% \mathrm{CI}=-0.79$ to 1.07$)$ were identified. When comparing the sharing and causal models, the $\Delta$ ELPD $>0$ $(\Delta \mathrm{ELPD}=0.022 ; \mathrm{SE}=0.835 ; p=0.51)$, indicating the posteriors from the causal model predict the data better, so the causal model may be a better fit (Supplementary Fig. 2).

At a nominally significant $p$ value threshold $(p<0.05)$, we detected causal effects of genetic liability to SCZ on very severe COVID (A2) and COVID-19 susceptibility (D1), of genetic liability to BIP on severe and very severe COVID-19 (A1 and B2), and of genetic liability to AD on COVID-19 susceptibility (C1 and D1, Supplementary Table 3A). In line with the significant GSMR finding for BIP-SCZ, all of these nominally significant results had positive odds ratios for (neuro)psychiatric disorders on COVID-19 phenotypes (Supplementary Table 3A; see Supplementary Table 3B for sensitivity analysis and Supplementary Table 4 for reverse results). The other four MR models' results were consistent with the GSMR findings (Supplementary Table 5). Sensitivity analyses using $p$ value $<10^{-7}$ to select more genetic instruments yielded consistent results, i.e., only genetic liability to BIPSCZ significantly increased risk to COVID-19, while liabilities to SCZ, BIP, and AD increased risk at nominally significant levels (Supplementary Table 3B).

Reverse MR results of COVID-19 phenotypes as potential risk factors of neuropsychiatric disorders

Three COVID-19 phenotypes met our predefined cut-off for inclusion as exposures, i.e., GWASs with $\geq 2$ genome-wide significant hits, in univariable MR analysis, 


\section{a}

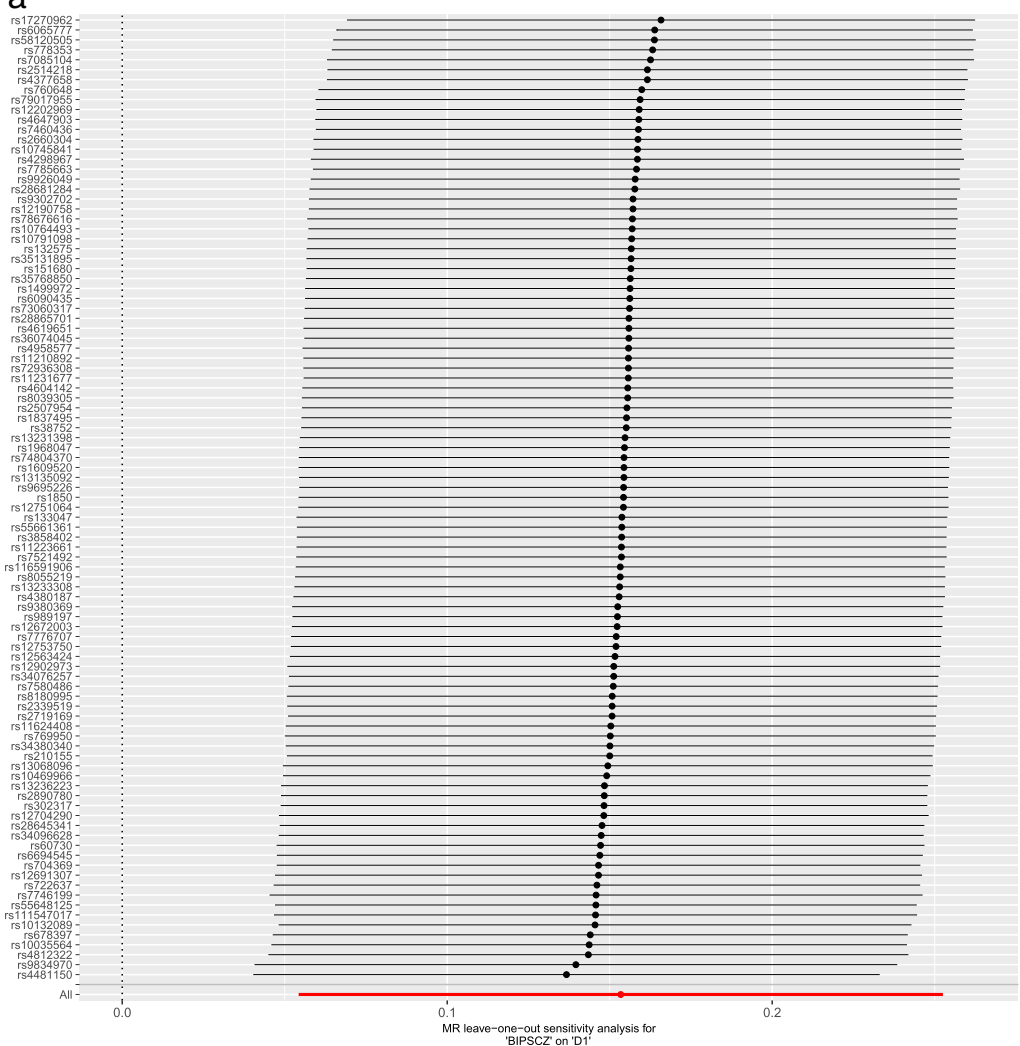

b

MR Method

Inverse variance weighted

MR Egger

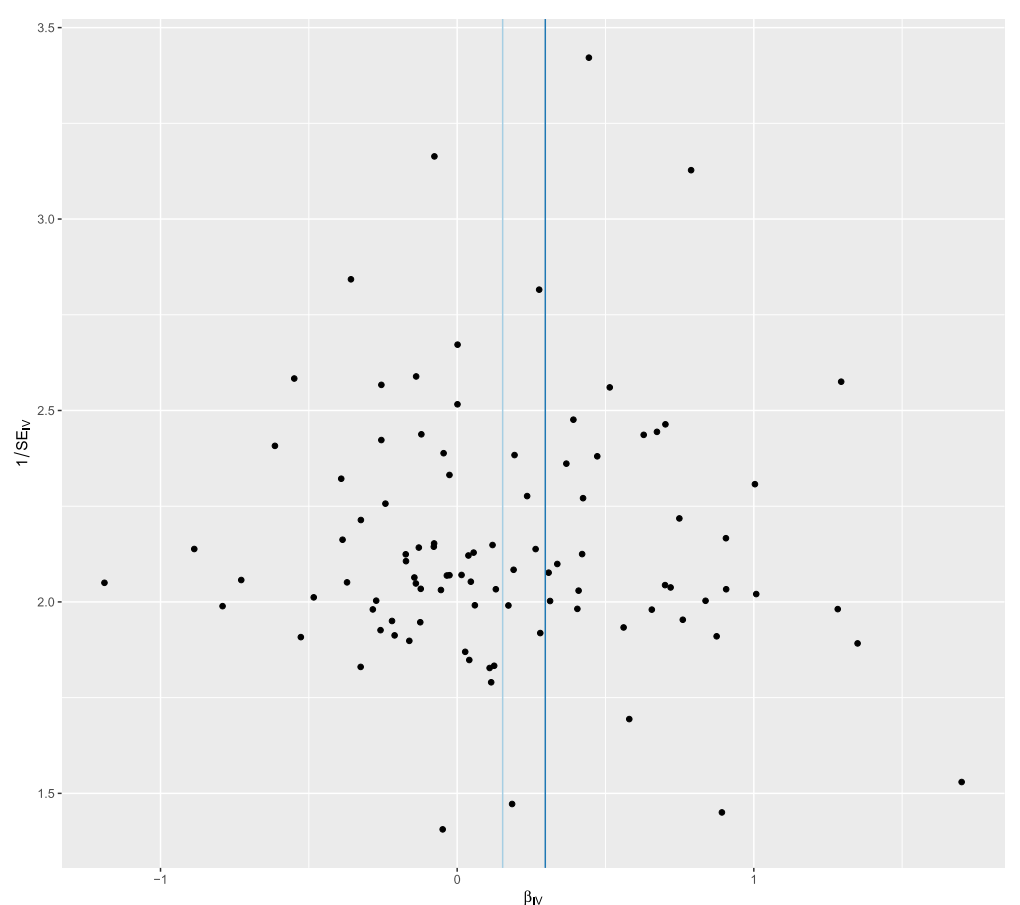

Fig. 2 (See legend on next page.) 
(see figure on previous page)

Fig. 2 Leave-one-out analysis and funnel plot of univariable bipolar-schizophrenia results. a Leave-one-out analysis to evaluate whether any single instrumental variable was driving the causal association of BIP-SCZ with self-reported COVID-19 disproportionately. As can be appreciated from the graph, no genetic variant altered the pooled beta coefficient, indicating the stability of our results. b Generalized funnel plot of univariable MR analysis of BIP-SCZ genetic liability effects on self-reported COVID-19 with first-order IMW and MR-Egger regression slopes to look for asymmetry as a sign of pleiotropy. This kind of graph plots the ratio estimate for each variant on the horizontal axis against its square-root precision (or weight) on the vertical axis. As can be appreciated from the plot, no evidence for asymmetry was detected, indicating the absence of directional pleiotropy and the instrument strength independent of direct effect (InSIDE) assumption to be satisfied.

namely severe and very severe COVID-19 (A2, B2) as well as COVID-19 susceptibility (C2, Table 2). MDD did not have enough overlapping SNPs to extract $\geq 2$ instrument variables, resulting in 18 tests performed in reverse analyses (six for A2, B2, and C2; Table 2; and Supplementary Table 4A). The $p$ value cut-off for significance was thus Bonferroni corrected to $0.05 / 18<0.0028$. We found no results withstanding this multiple-testing correction. Among most significant results were: genetic liability to very severe COVID-19 (A2) increasing risk of BIP-SCZ (GSMR OR, 1.036, 95\% CI, 1.002-1.071; $p=0.04$ ) and of SCZ (GSMR OR, 1.037, 95\% CI, 1.001-1.073; $p=0.042$; Supplementary Table 4A). The other four MR models' results were in line with the GSMR results (Supplementary Table 5). Sensitivity analyses using $p$ value $<10^{-7}$ to select more genetic instruments also yielded consistent results (Supplementary Table 4B).

\section{Multivariable MR analyses}

In forward MVMR, we examined the potential effects of genetic liabilities to three (neuro)psychiatric phenotypes jointly (AD, BIP, and SCZ; see "Methods" section) on seven COVID-19 phenotypes, resulting in a Bonferroni corrected (for the number of exposures) significance threshold of $p<0.05 / 7=0.0071$ (Supplementary Table 6A). No (neuro)psychiatric disorder showed a robust relationship with COVID-19 in MVMR-IVW models. Genetic liability to AD, BIP, and SCZ showed a nominally significant causal relationship with COVID-19. However, the estimates were not consistent with estimates from the MVMR-Egger sensitivity analysis, where no $p$ values were $<0.05$ (Supplementary Table 6A).

For reverse MVMR analyses examining the potential effects of genetic liabilities to three COVID-19 phenotypes jointly (A2, B2, and C2, i.e., GWASs with $\geq 2$ genome-wide significant hits) on (neuro)psychiatric phenotypes, no COVID-19 phenotypes showed a causal relationship with any of neuropsychiatric disorders at a Bonferronicorrected or nominal significance level in both IVW and MR-Egger (Supplementary Table 6B). The estimates were consistent with estimates from the MVMR-Egger sensitivity analyses (Supplementary Table 6B).

In sensitivity analysis \#2 using univariable GSMR analyses, we found BMI, EA, CP, and CAD had causal effects on COVID-19 sub phenotypes (Supplementary Table 7).
When adding all conditions (namely BMI, EA, CP, MDD, BIP-SCZ, CAD, CDG, BIP, SCZ, AD, and T2D) into MVMR models, causal effects were observed only between BMI and COVID-19 phenotypes (A2, B2, and C2). MVMR-IVW model results were consistent with MVMR-Egger, meaning no neuropsychiatric disorder showed a robust relationship with COVID-19 in these MVMR models (Supplementary Table 8).

In the end, we estimated genetic heritability and genetic correlations between (neuro)psychiatric phenotypes, COVID-19 (Table 1) and BMI. We show these in Supplementary Table 9. For (neuro)psychiatric phenotypes we found only nominally significant $(p<0.05)$ genetic correlations. At a Bonferroni-corrected significance threshold $(0.05 / 61=0.00082)$ we found only significant correlations between BMI and COVID-19 phenotypes. In addition, the LDSC intercepts were close to 0 , further confirming the absence of substantial sample overlap between GWASs in this MR study.

\section{Discussion}

We evaluated potential bidirectional associations between (neuro)psychiatric diseases and COVID-19 susceptibility and severity. While our univariable GSMR results hint that genetic liability to a combined phenotype of BIP and SCZ could slightly increase susceptibility to COVID-19, other methods including MVMR did not confirm this finding.

Our findings of no consistently increased risks of genetic liabilities to psychiatric disorders on COVID-19 are consistent with recent epidemiological observations in the UK and South Korea ${ }^{1,4}$ but inconsistent with other reports ${ }^{2,3,5}$. Similarly, contrary to one study ${ }^{2}$, we found no evidence of COVID-19 influencing risk of psychiatric disorders. Also in contrast to previous studies, we found no evidence of certain psychiatric disorders increasing risk to develop COVID-19, e.g., of anxiety disorders ${ }^{3,5}$. As pointed out by the authors of such studies, lack of repeated measures may have resulted in misclassification of important covariates resulting in invalid correction for some covariates in one or more of those studies. Moreover, residual confounding due to some unmeasured variables, such as population stratification may result in overestimations of effect sizes in observational studies. Furthermore, BMI was either not included at all or not 
included as a continuous covariate in studies reporting positive associations between psychiatric disorders and COVID-19 ${ }^{2,4}$. As BMI may be the strongest risk factor for COVID-19 susceptibility (as also confirmed here), it is of interest to correct for BMI as a continuous trait in upcoming epidemiological studies of COVID-19 and psychiatric disorders. Finally, our findings of mostly absent associations or in the event of suggestive associations odds ratios slightly above 1 are in disagreement with some observational studies but in line with MR studies of other COVID-19 risk factors ${ }^{6-8,40}$.

Strengths of our study include the integration of univariable and multivariable, bidirectional MR analyses using many instrument variables drawn from large GWASs. We also included a range of (neuro)psychiatric as well as COVID-19 phenotypes and validated our results across available MR methods. Furthermore, verification of primary GSMR findings with other MR methods helped elucidate consistency of findings. Finally, employing a multivariable in addition to a univariable approach is a strength. However, our results should also be interpreted in light of several limitations. First, a general concern in MR studies is risk of sample overlap. We minimized the chances of sample overlap between exposure datasets and outcome datasets by excluding UKBB populations from (neuro)psychiatric GWASs and by excluding 23andMe cohorts from COVID-19 datasets. We confirmed the lack of substantial sample overlap with LDSC intercepts. Nonetheless, cryptic relatedness and potential sample overlap between exposure and outcome GWASs may result in some degree of instrument bias. However, the F-statistics we found were all above 36, allaying major concerns about weak instrument bias. Another limitation directly follows from the availability of GWAS data. For some phenotypes, such as obsessive-compulsive disorder and anorexia nervosa, no large datasets excluding the UKBB were available at the time of analysis or writing. For MDD and SCZ, summary statistics of larger GWASs may become available in the coming year. Similarly, as COVID-19 GWAS sample sizes ramp up, statistical power in MR analysis may increase. We encourage researchers to repeat MR analyses on other phenotypes and to use such larger GWAS datasets once they become available. To that end, we have uploaded our code to Github (see "Data availability" section below). Possibly, increased statistical power in GWASs of psychiatric phenotypes and COVID-19 will in future elucidate more genetic associations and thus empower future MR analyses. On a similar note, non-whites are underrepresented in GWASs. As individuals of Asian and African ancestries seem particularly prone to develop COVID- $19^{41}$, we hope future GWAS encompassing multi-ethnic cohorts will allow for more inclusive MR analyses. Finally, inherent in a design where univariable as well as multivariable, bidirectional analyses are performed, a large number of statistical tests is performed, increasing the multiple-testing burden and statistical penalty.

In conclusion, while genetic liability to BIP and SCZ combined slightly increased COVID-19 susceptibility in univariable GSMR analysis, other MR methods and multivariable analyses could only confirm genetic underpinnings of BMI to be causally implicated in COVID-19 susceptibility. Thus, using MR we found no consistent proof of genetic liabilities to (neuro)psychiatric disorders contributing to COVID-19 liability or vice versa, which is in line with at least two observational studies. As BMI is strongly associated with psychiatric disorders as well as COVID-19, previously reported positive associations between psychiatric disorders and COVID-19 may have resulted from statistical models incompletely capturing BMI as a continuous covariate.

\section{Author details}

${ }^{1}$ Department of Psychiatry, UMC Utrecht Brain Center, Utrecht University, Utrecht, The Netherlands. ${ }^{2}$ Second opinion outpatient clinic, GGNet Mental Health, Warnsveld, The Netherlands. ${ }^{3}$ Department of Translational Neuroscience, UMC Utrecht Brain Center, Utrecht University, Utrecht, The Netherlands. ${ }^{4}$ Department of Preventive Medicine, Institute of Biomedical Informatics, Bioinformatics Center, School of Basic Medical Sciences, Henan University, Kaifeng, China

\section{Data availability}

The datasets we accessed to perform our analyses may be found in the publications that listed as references for the datasets used.

\section{Code availability}

We have made our code publicly available on Github (https://github.com/ Bochao1/MR_PSY_COVID19).

Conflict of interest

The authors declare no competing interests.

\section{Publisher's note}

Springer Nature remains neutral with regard to jurisdictional claims in published maps and institutional affiliations.

Supplementary information The online version contains supplementary material available at https://doi.org/10.1038/s41398-021-01325-7.

Received: 19 February 2021 Revised: 25 February 2021 Accepted: 19 March 2021

Published online: 08 April 2021

\section{References}

1. van der Meer, D. et al. Associations between psychiatric disorders, COVID-19 testing probability and COVID-19 testing results: findings from a populationbased study. BJPsych Open https://doi.org/10.1192/bjo.2020.75 (2020).

2. Taquet, M., Luciano, S., Geddes, J. R. \& Harrison, P. J. Bidirectional associations between COVID-19 and psychiatric disorder: a study of 62,354 COVID-19 cases. Lancet Psych. https://doi.org/10.1101/2020.08.14.20175190 (2020).

3. Yang, $H$. et al. Pre-pandemic psychiatric disorders and risk of COVID-19: a cohort analysis in the UK Biobank. Lancet Healthy Longevity https:/doi.org/ 10.1101/2020.08.07.20169847 (2020)

4. Lee, S. W. et al. Association between mental illness and COVID-19 susceptibility and clinical outcomes in South Korea: a nationwide cohort study. Lancet Psychiatry 7, 1025-1031 (2020). 
5. Wang, Q., Xu, R. \& Volkow, N. D. Increased risk of COVID-19 infection and mortality in people with mental disorders: analysis from electronic health records in the United States. World Psychiatry 20, 124-130 (2020).

6. Chadeau-Hyam, M. et al. Risk factors for positive and negative COVID-19 tests: a cautious and in-depth analysis of UK biobank data. Int. J. Epidemiol. 49, 1454-1467 (2020)

7. Ponsford, M. J. et al. Cardiometabolic Traits, Sepsis, and Severe COVID-19: A Mendelian Randomization Investigation. Circulation 142, 1791-1793 (2020).

8. Leong, A. et al. Cardiometabolic risk factors for COVID-19 susceptibility and severity: a mendelian randomization analysis. medRxiv e1003553 (2020).

9. Lin, B. D. et al. Assessing causal links between metabolic traits, inflammation and schizophrenia: a univariable and multivariable, bidirectional Mendelianrandomization study. Int J. Epidemiol. 48, 1505-1514 (2019).

10. Kunkle, B. W. et al. Genetic meta-analysis of diagnosed Alzheimer's disease identifies new risk loci and implicates Abeta, tau, immunity and lipid processing. Nat. Genet. 51, 414-430 (2019).

11. Otowa, T. et al. Meta-analysis of genome-wide association studies of anxiety disorders. Mol. Psychiatry 21, 1485 (2016).

12. Meier, S. M. et al. Genetic variants associated with anxiety and stress-related disorders: a genome-wide association study and mouse-model study. JAMA Psychiatry 76, 924-932 (2019).

13. Hyde, C. L. et al. Identification of 15 genetic loci associated with risk of major depression in individuals of European descent. Nat. Genet. 48, 1031-1036 (2016).

14. Stahl, E. A. et al. Genome-wide association study identifies 30 loci associated with bipolar disorder. Nat. Genet. 51, 793-803 (2019).

15. Pardinas, A. F. et al. Common schizophrenia alleles are enriched in mutationintolerant genes and in regions under strong background selection. Nat. Genet. 50, 381-389 (2018).

16. Bipolar D, Schizophrenia Working Group of the Psychiatric Genomics Consortium. Electronic address drve, Bipolar D, Schizophrenia Working Group of the Psychiatric Genomics C. Genomic dissection of bipolar disorder and schizophrenia, including 28 subphenotypes. Cell 173, 1705-15 e16 (2018).

17. Willer, C. J., Li, Y. \& Abecasis, G. R. METAL: fast and efficient meta-analysis of genomewide association scans. Bioinformatics 26, 2190-2191 (2010).

18. Scott, R. A. et al. An expanded genome-wide association study of type 2 diabetes in europeans. Diabetes 66, 2888-2902 (2017).

19. Initiative $\mathrm{C}-\mathrm{HG}$. The COVID-19 host genetics initiative, a global initiative to elucidate the role of host genetic factors in susceptibility and severity of the SARS-CoV-2 virus pandemic. Eur. J. Hum. Genet. 28, 715-718 (2020).

20. Zhu, Z. H. et al. Causal associations between risk factors and common diseases inferred from GWAS summary data. Nat. Commun. 9, 224.

21. Yang, J., Lee, S. H., Goddard, M. E. \& Visscher, P. M. GCTA: a tool for genomewide complex trait analysis. Am. J. Hum. Genet. 88, 76-82 (2011).

22. Burgess, S. \& Thompson, S. G. Bias in causal estimates from Mendelian randomization studies with weak instruments. Stat. Med. 30, 1312-1323 (2011).

23. Bowden, J., Davey Smith, G. \& Burgess, S. Mendelian randomization with invalid instruments: effect estimation and bias detection through Egger regression. Int J. Epidemiol. 44, 512-525 (2015).
24. Verbanck, M., Chen, C. Y., Neale, B. \& Do, R. Detection of widespread horizontal pleiotropy in causal relationships inferred from Mendelian randomization between complex traits and diseases. Nat. Genet. 50, 693-698 (2018).

25. Hemani, G. et al. The MR-Base platform supports systematic causal inference across the human phenome. Elife 7, e34408 (2018).

26. Morrison, J. et al. Mendelian randomization accounting for correlated and uncorrelated pleiotropic effects using genome-wide summary statistics. Nat. Genet 52, 740-747 (2020).

27. Burgess, S. \& Thompson, S. G. Multivariable Mendelian Randomization: the use of pleiotropic genetic variants to estimate causal effects. Am. J. Epidemiol. 181, 251-260 (2015).

28. Sanderson, E., Davey Smith, G., Windmeijer, F. \& Bowden, J. An examination of multivariable Mendelian randomization in the single-sample and two-sample summary data settings. Int J. Epidemiol. 48, 713-727 (2019).

29. Lim, K. X. et al. Studying individual risk factors for self-harm in the UK Biobank: a polygenic scoring and Mendelian randomisation study. PLoS Med. 17, e1003137 (2020).

30. Bulik-Sullivan, B. K. et al. LD score regression distinguishes confounding from polygenicity in genome-wide association studies. Nat. Genet. 47, 291-295 (2015)

31. Choi, K. W. et al. Assessment of bidirectional relationships between physical activity and depression among adults: a 2-sample Mendelian Randomization Study. JAMA Psychiatry 76, 399-408 (2019).

32. Hartwig, F. P. et al. Inflammatory biomarkers and risk of schizophrenia: a 2 sample Mendelian Randomization Study. JAMA Psychiatry 74, 1226-1233 (2017).

33. Gage, S. H. et al. Assessing causality in associations between cannabis use and schizophrenia risk: a two-sample Mendelian randomization study. Psychol. Med. 47, 971-980 (2017).

34. Locke, A. E. et al. Genetic studies of body mass index yield new insights for obesity biology. Nature 518, 197-206 (2015)

35. Nelson, C. P. et al. Association analyses based on false discovery rate implicate new loci for coronary artery disease. Nat. Genet. 49, 1385-1391 (2017).

36. Lee, J. J. et al. Gene discovery and polygenic prediction from a genome-wide association study of educational attainment in 1.1 million individuals. Nat. Genet. 50, 1112-1121 (2018).

37. Cross-Disorder Group of the Psychiatric Genomics Consortium. Electronic address pmhe, cross-disorder group of the psychiatric genomics C. genomic relationships, Novel Loci, and pleiotropic mechanisms across eight psychiatric disorders. Cell 179, 1469-82 e11 (2019).

38. Howard, D. M. et al. Genome-wide meta-analysis of depression identifies 102 independent variants and highlights the importance of the prefrontal brain regions. Nat. Neurosci. 22, 343-352 (2019).

39. Purves, K. L. et al. A major role for common genetic variation in anxiety disorders. Mol. Psychiatry 25, 3292-3303 (2020).

40. Zhou, Y. et al. Coagulation factors and COVID-19 severity: Mendelian randomization analyses and supporting evidence. medRxiv https://doi.org/10.1101/ 2020.11.20.20235440 (2020).

41. Sze, S. et al. Ethnicity and clinical outcomes in COVID-19: a systematic review and meta-analysis. E Clini. Med. 29, 100630 (2020). 